\title{
Task-Switching Performance With 1:1 and 2:1 Cue-Task Mappings: Not So Different After All
}

\author{
Darryl W. Schneider \\ Carnegie Mellon University and Vanderbilt University
}

\author{
Gordon D. Logan \\ Vanderbilt University
}

\begin{abstract}
When task-switching studies use the task-cuing procedure with a 1:1 cue-task mapping, task switching and cue switching are confounded, which is problematic for interpreting switch costs. The use of a 2:1 cue-task mapping is a potential solution to this problem, but it is possible that introducing more cues may also introduce marked changes in task-switching performance. In 5 experiments involving 160 subjects, the authors compared performance with 1:1 and 2:1 mappings across several methodological changes. Differences in switch costs between mappings were small and, in most analyses, nonsignificant. In all experiments, both mappings yielded significant reductions in switch cost across cue-target interval, and there were significant cue-switching effects with the 2:1 mapping. A model of cue encoding fit the data from both mappings about equally well. Overall, task-switching performance was more similar than it was different between mappings, leading the authors to suggest that the use of a 2:1 mapping is a viable solution to the problem associated with a 1:1 mapping.
\end{abstract}

Keywords: task switching, cues, cue-task mapping, switch cost, sequential effects

There is a problem in the world of task-switching research. The problem occurs in the task-cuing procedure (Meiran, 1996; Sudevan $\&$ Taylor, 1987), in which a cue is presented on each trial that indicates a task to perform on a target. For example, the cues Parity and Magnitude could indicate that a target digit should be judged as odd/even or lower/higher than 5, respectively. Cues appear in random order across trials, resulting in task switches (e.g., Parity to Magnitude) and task repetitions (e.g., Magnitude to Magnitude). Task switches are slower than task repetitions, and changes in these switch costs with experimental manipulations have been studied extensively (Monsell, 2003). One manipulation afforded by the task-cuing procedure is variation of the cue-target interval (CTI). By manipulating CTI and observing the effect on switch cost, one can investigate processing associated with cue encoding, task identification, and task preparation. This is a key advantage of the task-cuing procedure as a task-switching methodology (Logan \& Bundesen, 2003; Logan, Schneider, \& Bundesen, 2007; Mayr \& Kliegl, 2003; Meiran, 1996; Meiran, Chorev, \& Sapir, 2000; Monsell \& Mizon, 2006).

An assumption underlying inferences about switch costs is that they reflect the act of task switching or a consequence of it.

This article was published Online First February 7, 2011.

Darryl W. Schneider, Department of Psychology, Carnegie Mellon University and Department of Psychology, Vanderbilt University; Gordon D. Logan, Department of Psychology, Vanderbilt University.

This research was supported by National Institute of Mental Health Grant R01-MH073878-01 awarded to Gordon D. Logan. We are grateful to Julie Delheimer for running all the subjects in the experiments. We thank Iring Koch, Michael Masson, and Stephen Monsell for their comments on previous versions of this article.

Correspondence concerning this article should be addressed to Darryl W. Schneider, Department of Psychology, Carnegie Mellon University, Pittsburgh, PA 15213. E-mail: dws@cmu.edu
However, when a 1:1 cue-task mapping is used in the task-cuing procedure, as in the earlier example and in many studies (e.g., Altmann, 2004; Arrington, Altmann, \& Carr, 2003; Koch, 2008; Meiran, 1996; Meiran et al., 2000), this assumption is not completely valid because task switching is confounded with cue switching: Whenever the task switches, the cue switches; whenever the task repeats, the cue repeats. This is a problem because it means that switch costs could reflect task switching, cue switching, or both. For example, there might be a task-switching component due to a cost of task-set reconfiguration (Monsell \& Mizon, 2006) and a cue-switching component due to a benefit of encoding a repeated cue (Logan \& Bundesen, 2003). One component or the other might comprise most of the switch cost, but with a $1: 1$ mapping their relative contributions cannot be determined. Given that researchers usually want to make inferences about task switching, it is essential that there may be some way to distinguish its effects from those of cue switching.

A potential solution to the problem is to use a 2:1 cue-task mapping (Logan \& Bundesen, 2003; Mayr \& Kliegl, 2003). For example, the cues Parity and Odd-Even could indicate an odd/ even judgment and the cues Magnitude and Low-High could indicate a lower/higher than 5 judgment. This partially deconfounds task switching and cue switching because it produces three kinds of transitions: task switches (task and cue both switch; e.g., Magnitude to Parity), task repetitions (task repeats but cue switches; e.g., OddEven to Parity), and cue repetitions (task and cue both repeat; e.g., Parity to Parity). By comparing task switches with task repetitions, one can examine task-switching effects with a constant cue transition. By comparing task repetitions with cue repetitions, one can examine cue-switching effects with a constant task transition. These comparisons have been done many times (e.g., Logan \& Bundesen, 2003; Mayr \& Kliegl, 2003; Monsell \& Mizon, 2006; for a summary of several studies, see Logan et al., 2007) and a consistent pattern has emerged: Task switches are slower than task 
Table 1

Differences in Experiment Design and Switch Costs by Mapping

\begin{tabular}{|c|c|c|c|c|c|c|c|c|}
\hline \multirow[b]{2}{*}{ Experiment } & \multirow[b]{2}{*}{$N$} & \multirow[b]{2}{*}{ Uncued trials } & \multirow[b]{2}{*}{ Cues } & \multirow[b]{2}{*}{ Tasks } & \multirow[b]{2}{*}{ CTIs } & \multicolumn{3}{|c|}{ Switch cost } \\
\hline & & & & & & 2:1 mapping & $1: 1$ mapping & Difference \\
\hline 1 & 32 & No & Words & Magnitude/Parity & 5 & $85(2.0)$ & $71(1.7)$ & $14(0.3)$ \\
\hline 2 & 32 & Yes & Words & Magnitude/Parity & 5 & $116(1.8)$ & $110(1.4)$ & $6(0.4)$ \\
\hline 3 & 32 & Yes & Letters & Magnitude/Parity & 5 & $188(0.9)$ & $172(1.2)$ & $16(-0.3)$ \\
\hline 4 & 32 & Yes & Letters & Height/Width & 5 & $222(1.8)$ & $170(2.3)$ & $52^{*}(-0.5)$ \\
\hline 5 & 32 & Yes & Letters & Height/Width & 2 & $238(1.2)$ & $208(1.5)$ & $30(-0.3)$ \\
\hline Altmann (2006) & 15 & Yes & Letters & Height/Width & 2 & 360 & 194 & $166^{*}$ \\
\hline
\end{tabular}

Note. $\quad N=$ number of subjects with data for each mapping; CTI = cue-target interval. Numbers in parentheses are percentage error rates. Switch costs are in milliseconds.

${ }^{*} p<.05$ for the Mapping $\times$ Transition interaction from the relevant analysis of variance. (For Altmann's data, we indicate the results of his statistical analyses.)

repetitions, whereas task repetitions are slower than cue repetitions. This suggests that switch costs obtained with a 1:1 mapping (reflecting differences between task switches and cue repetitions) are composed of task-switching and cue-switching effects, creating an inferential problem.

The issue addressed in the present study is whether the use of a 2:1 mapping is a viable solution to the problem. On the one hand, it enables fractionation of switch costs into task-switching and cue-switching effects that can each be measured and modulated (e.g., Arrington, Logan, \& Schneider, 2007; Mayr \& Kliegl, 2003). On the other hand, introducing more cues may also introduce marked changes in performance. For example, switch costs might differ between 1:1 and 2:1 mappings. Indeed, Altmann (2006) found that switch cost was much larger with a 2:1 mapping (360 ms) compared with a 1:1 mapping (194 ms). This finding suggests that there may be important differences between mappings (cf. Mayr, in press), but one should be cautious when drawing conclusions from a single experiment.
Our goal was to determine whether performance differs between $1: 1$ and 2:1 mappings in a systematic comparison of the two conditions. We conducted five experiments in which the mappings were manipulated within subjects in separate sessions. Experiment 1 was based on the design of previous experiments from our laboratory: All trials were cued, the cues were words, the tasks were magnitude and parity judgments of digits, and there were five CTIs. Altmann's (2006) experiment differed in several respects: Every second trial was an uncued task repetition, the cues were letters, the tasks were height and width judgments of rectangles, and there were two CTIs. To assess the importance of these differences, we progressively changed our design across experiments to make it closer to Altmann's (see Table 1). Each experiment was a replication of its precursor with one major change. In Experiment 2, every second trial was an uncued task repetition. In Experiment 3, the cues were letters. In Experiment 4, the tasks were height and width judgments of rectangles. In Experiment 5, there were two CTIs.

Table 2

Summary of Analyses of Variance

\begin{tabular}{|c|c|c|c|c|c|c|c|c|c|c|c|c|c|c|c|c|c|c|}
\hline \multirow[b]{2}{*}{ Effect } & \multicolumn{5}{|c|}{ Cued response time } & \multicolumn{5}{|c|}{ Cued error rate } & \multicolumn{4}{|c|}{ Uncued response time } & \multicolumn{4}{|c|}{ Uncued error rate } \\
\hline & 1 & 2 & 3 & 4 & 5 & 1 & 2 & 3 & 4 & 5 & 2 & 3 & 4 & 5 & 2 & 3 & 4 & 5 \\
\hline Order $(\mathrm{O})$ & & & & & & & & & & & & & * & & & & & \\
\hline Mapping (M) & & $*$ & & & & & $*$ & & & * & & & & & & & & \\
\hline $\mathrm{O} \times \mathrm{M}$ & * & $*$ & * & * & $*$ & & * & & $*$ & & $*$ & * & * & $*$ & $*$ & $*$ & $*$ & * \\
\hline Transition (T) & * & * & * & * & * & $*$ & * & $*$ & $*$ & $*$ & & & & $*$ & $*$ & & & * \\
\hline $\mathrm{O} \times \mathrm{T}$ & & & & & & & & & & & & & & & & & & \\
\hline $\mathrm{M} \times \mathrm{T}$ & & & & * & & & & & & & & & & & & & & \\
\hline $\mathrm{O} \times \mathrm{M} \times \mathrm{T}$ & * & & * & * & * & & * & * & & * & & & & & & & & \\
\hline CTI (C) & * & * & * & * & * & & * & & * & * & & & & * & & & & \\
\hline $\mathrm{O} \times \mathrm{C}$ & & & $*$ & & & & & & & & * & & & & & & & \\
\hline $\mathrm{M} \times \mathrm{C}$ & & & * & $*$ & $*$ & & & & & & & & & & & & & \\
\hline $\mathrm{O} \times \mathrm{M} \times \mathrm{C}$ & * & * & * & * & * & & & & & & & & & & & & & \\
\hline $\mathrm{T} \times \mathrm{C}$ & * & * & * & * & $*$ & & & & & & & & & & & & & * \\
\hline $\mathrm{O} \times \mathrm{T} \times \mathrm{C}$ & & & & & & & & & & & & & & & & & & \\
\hline $\mathrm{M} \times \mathrm{T} \times \mathrm{C}$ & & & & & & & & & & & & & & * & & & & \\
\hline $\mathrm{O} \times \mathrm{M} \times \mathrm{T} \times \mathrm{C}$ & & & & & & & & & & & & & & & & * & & \\
\hline
\end{tabular}

Note. Numbers in column headers refer to experiments. CTI $=$ cue-target interval. Complete results for all analyses of variance are available upon request from Darryl W. Schneider.

${ }^{*} p<.05$. 

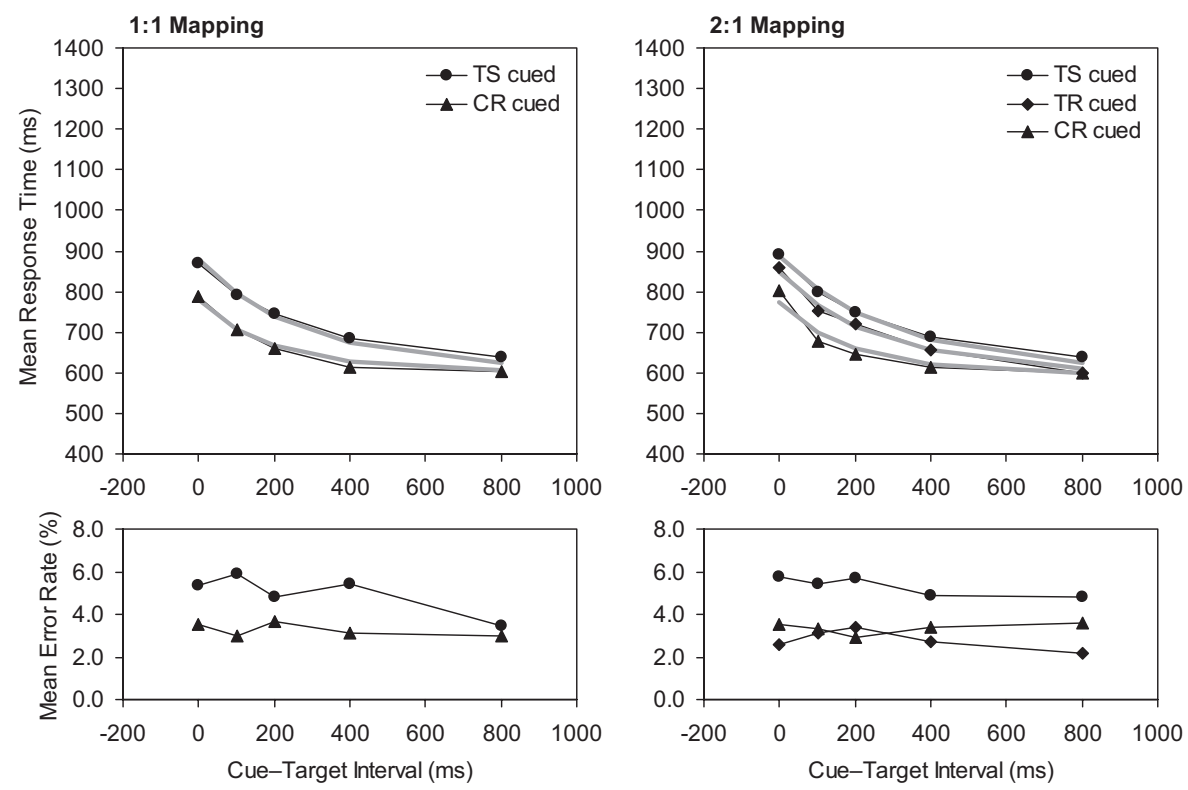

Figure 1. Mean response time and error rate in Experiment 1 as a function of mapping, transition $(\mathrm{TS}=\operatorname{task}$ switch; $\mathrm{TR}=$ task repetition; $\mathrm{CR}=$ cue repetition), and cue-target interval. Points $=$ experiment data; thick gray lines $=$ model predictions.

\section{Method}

\section{Subjects}

One hundred sixty students from Vanderbilt University each completed two sessions for monetary compensation. There were 32 subjects per experiment. Half the subjects had a 1:1 mapping in the first session and a 2:1 mapping in the second session; the other subjects had the reverse order.

\section{Materials}

In Experiments 1-3, the tasks were magnitude (lower/higher than 5) and parity (odd/even) judgments of target digits (1-9, excluding 5). In Experiments 1 and 2, the words Magnitude and Low-High cued the magnitude task and the words Parity and Odd-Even cued the parity task. Category order in the Low-High and $O d d-E v e n$ cues was spatially compatible with each subject's category-response assignments. In Experiment 3, the letters $M$ and $U$ cued the magnitude task, and the letters $P$ and $K$ cued the parity task. In Experiments 4 and 5, the tasks were height (short/tall) and width (thin/thick) judgments of rectangles (a set of four grayoutlined rectangles representing all combinations of side lengths 3.5 and $7.0 \mathrm{~cm}$ ). The letters $H$ and $U$ cued the height task and the letters $W$ and $L$ cued the width task. All text stimuli were displayed in white on a black screen and viewed from about $50 \mathrm{~cm}$.

\section{Procedure}

Each mapping was experienced in a separate session. In the 1:1 mapping session, there was only one cue per task in each block. Half the subjects had the cues Magnitude and Parity (Experiments 1 and 2), $M$ and $P$ (Experiment 3), or $H$ and $W$ (Experiments 4 and 5 ) in the first half of the session and the complementary pair of cues in the second half; the other subjects had the reverse order. When they reached the halfway point, subjects received instructions signaling the cue change. In the 2:1 mapping session, there were two cues per task in every block.

Each trial in a block began with either a 500-ms fixation display (two vertically aligned crosses in Experiments 1-3 or one cross in Experiment 4) or no fixation display (Experiment 5). A cue was then presented centrally, replacing the top cross in Experiments 1-3 or the lone cross in Experiment 4. After a CTI (0, 100, 200, 400, or $800 \mathrm{~ms}$ in Experiments 1-4 or 100 or $900 \mathrm{~ms}$ in Experiment 5), a target was presented, replacing the bottom cross in Experiments 1-3 or surrounding the cue in Experiments 4 and 5. Cue and target remained visible until a response, then the screen was cleared for $500 \mathrm{~ms}$. In Experiment 1, the next trial commenced immediately thereafter. In Experiments 2-5, a new target was presented at the same location as the previous target. Subjects were instructed to perform the previously cued task on the new target. After a response on this uncued trial, the screen was cleared for $500 \mathrm{~ms}$ and the next cued trial commenced thereafter, except in Experiment 5, where the response-cue interval (RCI) was either 900 or $100 \mathrm{~ms}$ depending on whether the CTI was 100 or $900 \mathrm{~ms}$, respectively (the RCI and CTI always summed to $1,000 \mathrm{~ms}$ ). The RCI was always 1,000 ms (500-ms blank screen plus 500-ms fixation display) in Experiments $1-4 .{ }^{1}$ Responses were made with the $Z$ and slash keys on a QWERTY keyboard, with same-task categories assigned to different keys and category-response assignments counterbalanced across subjects. Reminders of the

\footnotetext{
${ }^{1}$ Previous research has shown that carryover effects between trials are asymptotic at RCIs of 1,000 ms or longer (Meiran et al., 2000) and that CTI effects can be found separately of RCI effects (e.g., Logan \& Bundesen, 2003; Mayr \& Kliegl, 2003; Meiran, 1996; Meiran et al., 2000).
} 

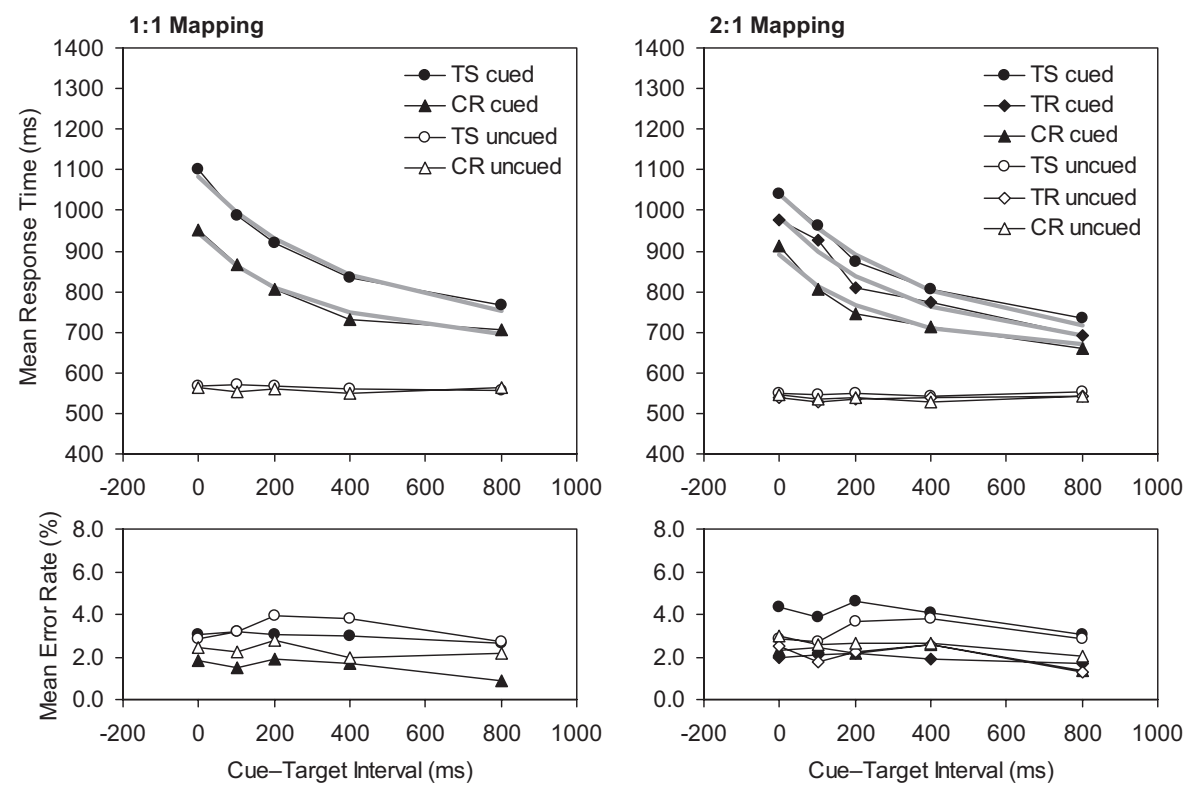

Figure 2. Mean response time and error rate in Experiment 2 as a function of mapping, transition (TS $=$ task switch; TR $=$ task repetition; $\mathrm{CR}=$ cue repetition), and cue-target interval. Points $=$ experiment data; thick gray lines $=$ model predictions.

category-response assignments appeared in the bottom corners of the screen during the experiment. Subjects were instructed to respond quickly and accurately.

Subjects completed 14, 8, 8, 8, and 10 blocks with 80, 160, 160, 160, and 128 trials per block in each session of Experiments 1-5, respectively. Half the trials in each block of Experiments 2-5 were cued and half were uncued. Cued trials were randomly selected from the full set of Cue $\times$ Target $\times$ CTI combinations associated with the relevant session in each experiment. All combinations in the set occurred equally often. The targets for uncued trials were randomly selected (independently of the targets for cued trials) from the full set of targets.

\section{Results and Discussion}

The first block of each half in the 1:1 mapping session and the first two blocks in the 2:1 mapping session were treated as practice. $^{2,3}$ We excluded the first trial of each block and trials with response times (RTs) exceeding three standard deviations of a subject's cell mean for the analysis of variance (ANOVA) design reported below. RT trimming with separate cutoffs for cued and uncued trials resulted in the exclusion of less than $3.0 \%$ of trials in each experiment. We included only correct trials in the RT analysis. We analyzed uncued trials only if the immediately preceding cued trials were correct. We submitted the RT and error data to ANOVAs, with mapping order as a between-subjects factor and mapping, transition, and CTI as within-subjects factors. ${ }^{4}$ The ANOVA results are summarized in Table 2. Error rates were $4.2 \%$, $2.7 \%(2.8 \%), 2.2 \%(2.1 \%), 3.0 \%(2.9 \%)$, and $2.5 \%(2.3 \%)$ for cued (uncued) trials in Experiments 1-5, respectively. The error patterns do not contradict the RT patterns (see Figures 1-5), so we focus on the latter.

\section{Do Switch Costs Differ Between Mappings?}

Figures 1-5 show mean cued RTs for Experiments 1-5, respectively. Switch costs (defined for both mappings as differences between task switches and cue repetitions) are summarized in Table 1. Differences in switch costs were small and nonsignificant except in Experiment 4. When all experiments were analyzed together in an ANOVA with experiment as a factor, the overall $24-\mathrm{ms}$ difference in switch costs was significant $(p<.05)$. All of our differences were significantly smaller $(p<.001)$ than the 166-ms difference observed by Altmann (2006), even though we determined with $\mathrm{G}^{*}$ Power 3 (Faul, Erdfelder, Lang, \& Buchner, 2007) that the power to detect a difference of at least $100 \mathrm{~ms}$ exceeded .90 for each experiment. These results indicate that differences in switch costs between mappings are smaller and less robust than what Altmann found.

There was evidence that exposure to one mapping affected subsequent performance with the other, as indicated by signif-

\footnotetext{
${ }^{2}$ By changing the cues halfway through the 1:1 mapping session and excluding the first block of each half of that session as practice, the overall frequencies with which specific cues were experienced in the 1:1 and 2:1 mapping sessions, both in practice and in the experimental blocks, were equated.

${ }^{3}$ Due to computer errors, the data from the last block of the 2:1 mapping session for one subject in Experiment 3 and the data from the last 46 trials of the 2:1 mapping session for one subject in Experiment 4 were lost; however, there was still ample data for these subjects from earlier trials.

${ }^{4} \mathrm{We}$ also analyzed the data as a function of cue type. The main finding was that switch costs in Experiments 3-5 were smaller for cues representing the first letters of task names than for cues representing other letters.
} 

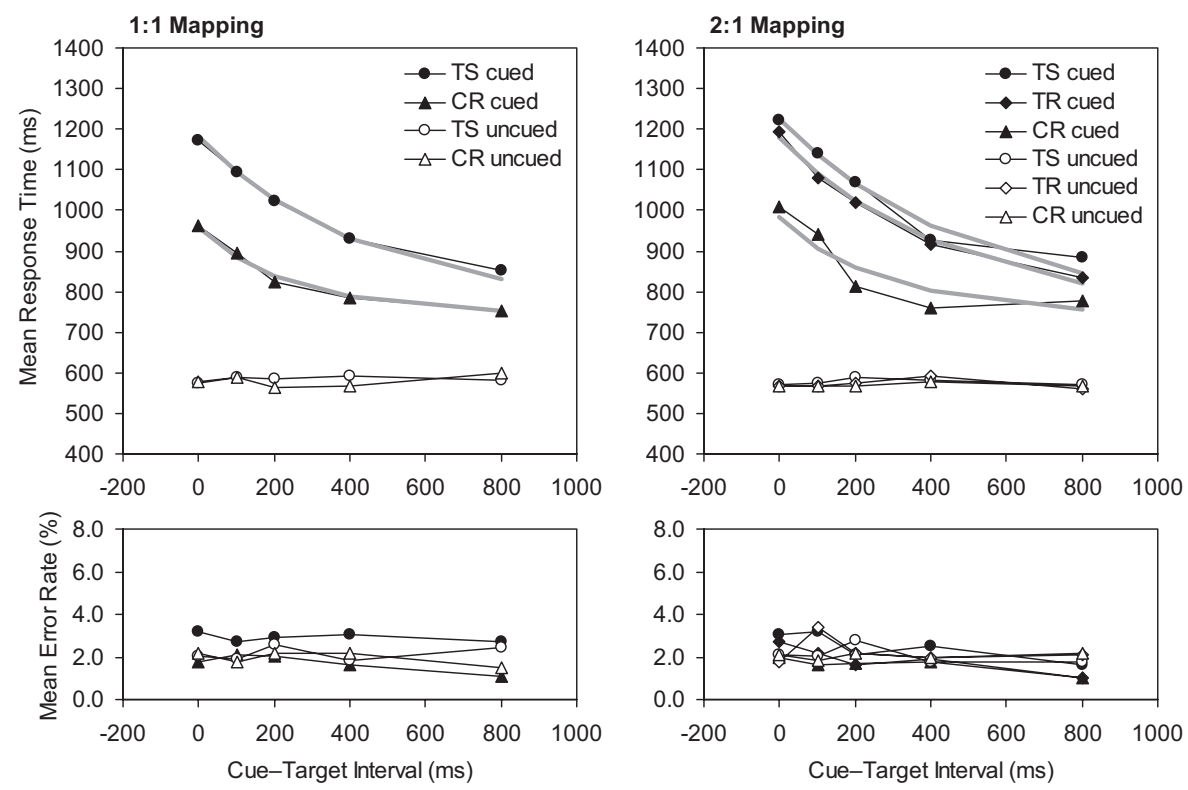

Figure 3. Mean response time and error rate in Experiment 3 as a function of mapping, transition $(\mathrm{TS}=\operatorname{task}$ switch; $\mathrm{TR}=$ task repetition; $\mathrm{CR}=$ cue repetition), and cue-target interval. Points $=$ experiment data; thick gray lines $=$ model predictions.

icant interactions involving mapping order (see Table 2). However, the interactions were not systematic across experiments (see Table 3), and additional analyses revealed that our conclusions are not compromised by these carryover effects. Separate ANOVAs on the data from each session (with mapping as a between-subjects factor) indicated no significant differences in switch costs in either the first or the second session. This was also true for first-session analyses restricted to data from the first half of the 1:1 mapping session, in which subjects had yet to experience the complementary pair of cues (see Table 3). ${ }^{5}$ The sizable numerical differences in Experiments 4 and 5 could be interpreted (if the lack of statistical significance is ignored) as suggesting that performance differs between mappings when the tasks are height and width judgments and subjects have not had extensive practice.

\section{Do Switch Costs Decrease Across CTI?}

Figures 1-5 and Table 4 show that switch costs decreased across CTI with both mappings in every experiment. ${ }^{6}$ These results differ from those of Altmann (2006), who found nonsignificant reductions in his experiment and concluded that the interaction was inconsistent.

\section{Are There Separate Task-Switching and Cue- Switching Effects?}

Figures $1-5$ and Table 5 show data for the three transitions that occur with a 2:1 mapping. Task switches were slower than task repetitions, indicating a task-switching effect, and task repetitions were slower than cue repetitions, indicating a cueswitching effect. There was a significant main effect of transition in each experiment $(p<.001)$, and contrasts indicated that both effects were significant in every experiment $(p<.05)$ except for the task-switching effect in Experiment $3(p=.12)$. These results indicate that there are robust task-switching and cue-switching effects with a 2:1 mapping. It seems reasonable to assume that both effects also occur with a 1:1 mapping, except they are conflated.

\section{Are There Uncued Switch Costs?}

Figures 2-5 show data for uncued trials. Following Altmann (2006), we coded the transition for an uncued trial based on the immediately preceding cued trial (e.g., if a cued trial was a task switch, then the following uncued trial was coded as a task switch even though it was actually a repetition of the cued task). This coding scheme allows one to assess carryover "switch costs" uncontaminated by cue processing. Uncued switch costs were 7, 7, 3, and $23 \mathrm{~ms}$ in Experiments 2-5, respectively, and only the last effect-which came from the experiment closest in design to Altmann's-was significant (see Table 2) and similar to the 34-ms switch cost found by Altmann. These results suggest that any

\footnotetext{
${ }^{5}$ The first-session data analyses with mapping as a between-subjects factor provide results that may be more suitable for comparison with those of Altmann's (2006) experiment, in which mapping was a betweensubjects variable.

${ }^{6}$ As shown in Table 5, the reduction in switch cost across CTI with the 2:1 mapping is largely due to a reduction in the cue-switching effect rather than the task-switching effect, suggesting that the latter is not strongly modulated by CTI (Mayr \& Kliegl, 2003; but see Monsell \& Mizon, 2006). One could infer that the reduction in switch cost across CTI with the $1: 1$ mapping largely reflects a reduction in the latent cue-switching component of the cost.
} 

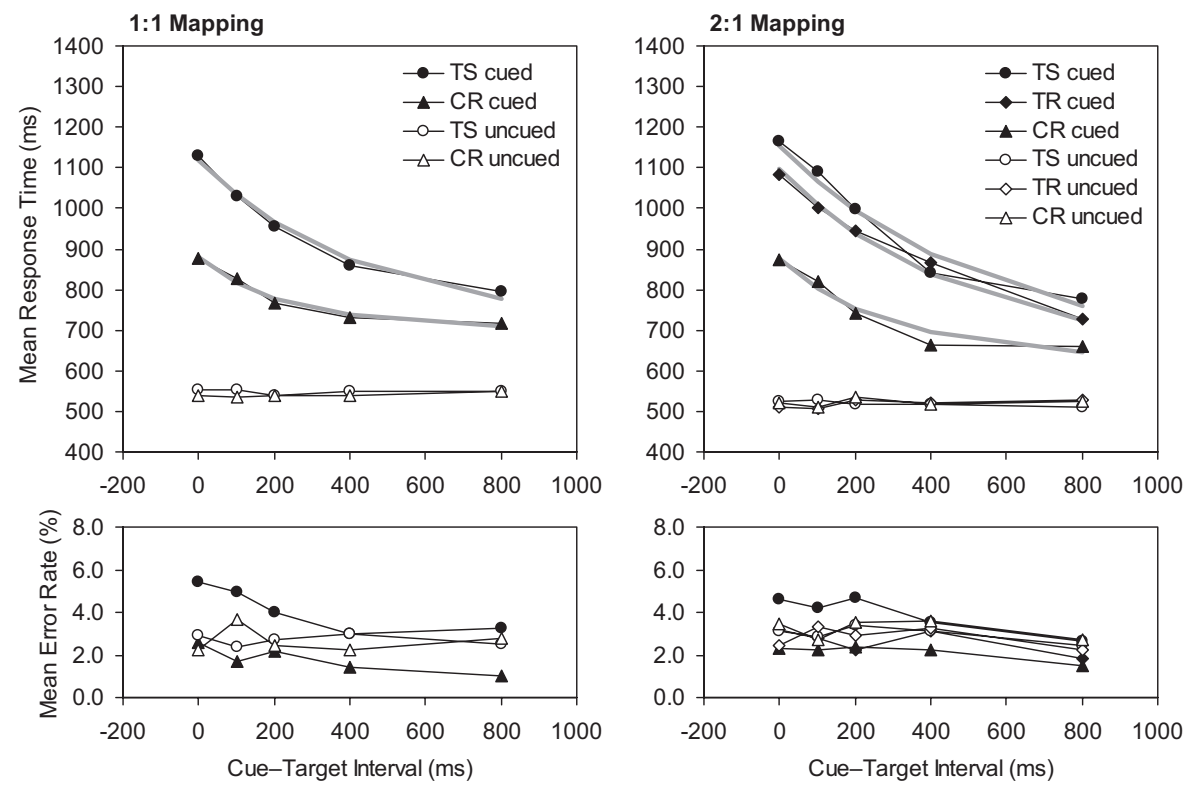

Figure 4. Mean response time and error rate in Experiment 4 as a function of mapping, transition $(\mathrm{TS}=$ task switch; $\mathrm{TR}=$ task repetition; $\mathrm{CR}=$ cue repetition), and cue-target interval. Points $=$ experiment data; thick gray lines $=$ model predictions.

carryover effects from unpredictable cued switching to predictable uncued repetition were minimal.

\section{Modeling}

In this section, we assess whether our model of cue encoding (Logan \& Bundesen, 2003; Schneider \& Logan, 2005) fits the data from both mappings about equally well. This is an important issue because Altmann (2006) obtained better fits of our model to 2:1 mapping data than to 1:1 mapping data, which suggested to him that the model had limited generality. Our model assumes that cued RT reflects the sum of cue encoding time (i.e., the time required to form a categorical representation of the cue; Arrington et al., 2007) and residual processing time (i.e., the time required for processes such as target encoding, response selection, and response execution; Schneider \& Logan, 2005, 2009). Cue encoding time is assumed to be exponentially distributed, with its contribution to RT decreasing as CTI becomes longer because cue encoding can occur during the CTI. This time-course function is represented by the equation:

$$
R T=R T_{\text {Base }}+\mu \cdot \exp (-C T I / \mu),
$$

where $R T_{\text {Base }}$ is residual processing time, and $\mu$ is mean cue encoding time. Note there is nothing inherent in Equation 1 that would favor 2:1 mapping data over 1:1 mapping data. This makes Altmann's finding of a difference in model fits all the more compelling and worthwhile investigating.

We fit Equation 1 to the individual-subject data and group data from each experiment. There was a separate $R T_{\text {Base }}$ for each mapping, and a separate $\mu$ for each transition (task switch or cue repetition) within each mapping, for a total of six free parameters. ${ }^{7}$ Parameters were optimized to minimize the rootmean-square deviation (RMSD) between data and model. The fit indices and best-fitting parameter values are summarized in Tables 6 and 7, respectively, and model predictions are plotted as gray lines in Figures 1-5. Figure 6 shows individual-subject fit indices for the 1:1 and 2:1 mapping fits plotted against one another. Although there is variability in the quality of the fits, there is no trend for better fits to $2: 1$ mapping data than to $1: 1$ mapping data. Out of 160 subjects, $43.1 \%$ had numerically smaller RMSD values, and $48.1 \%$ had numerically larger correlations for their $2: 1$ mapping fits compared with their $1: 1$ mapping fits.

Table 7 shows that there were some differences in best-fitting parameter values between mappings. For the fits to Experiments $1-3$, the $95 \%$ confidence intervals around the parameter values for the 1:1 mapping fits overlap those for the 2:1 mapping fits, suggesting no substantial differences. However, for the fits to

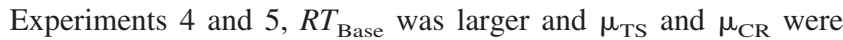
smaller for the 1:1 mapping fits compared with the 2:1 mapping fits (cf. Altmann, 2006). The group fits mirrored the individualsubject fits (see Tables 6 and 7), with the group parameter values falling within the $95 \%$ confidence intervals of the individualsubject parameter values.

\section{General Discussion}

When task-switching studies use the task-cuing procedure with a 1:1 cue-task mapping, task switching and cue switching are confounded, which is problematic for interpreting switch

\footnotetext{
${ }^{7}$ Altmann (2006) fit this model to his data but excluded task repetitions with the 2:1 mapping. To allow for direct comparison with his results, we do the same in our fits, although we also report fits that included task repetitions.
} 

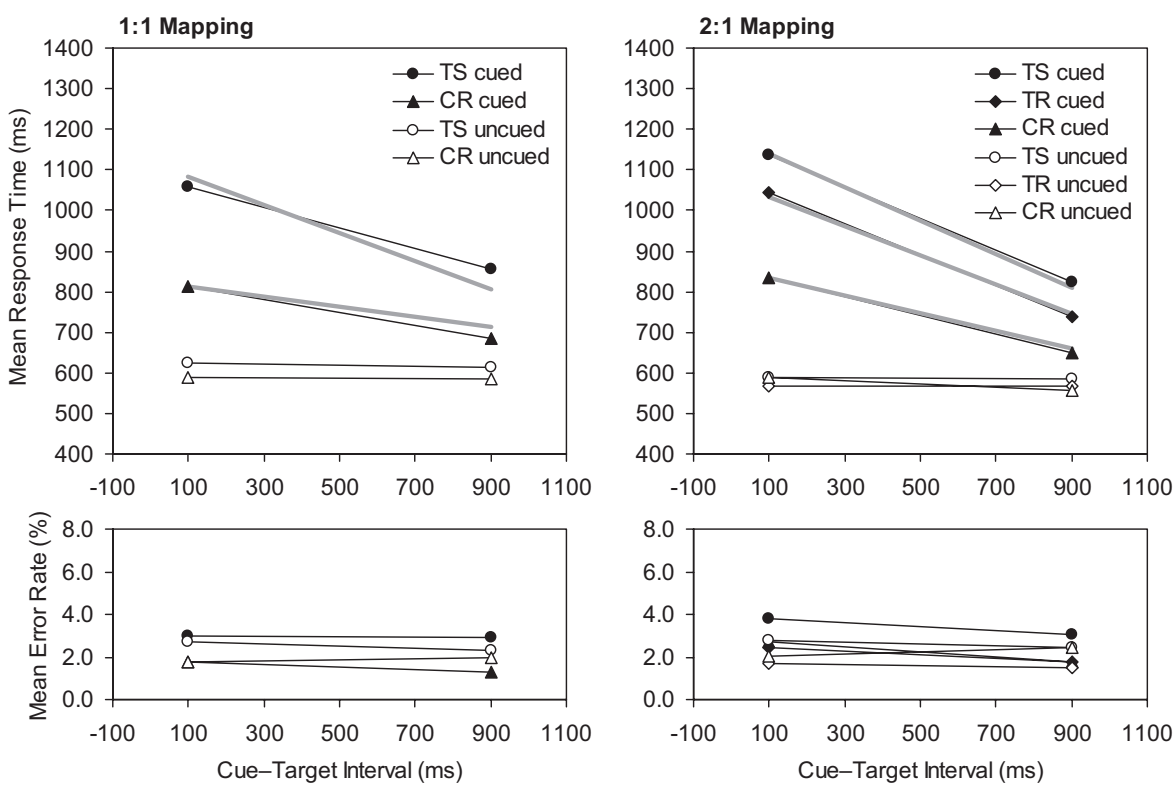

Figure 5. Mean response time and error rate in Experiment 5 as a function of mapping, transition $(\mathrm{TS}=\operatorname{task}$ switch; $\mathrm{TR}=$ task repetition; $\mathrm{CR}=$ cue repetition), and cue-target interval. Points $=$ experiment data; thick gray lines $=$ model predictions.

costs. The use of a 2:1 cue-task mapping is a potential solution to this problem, but it is possible that introducing more cues may also introduce marked changes in performance. In five experiments, we compared performance with 1:1 and 2:1 mappings across variations in trial cuing, cues, tasks, and number of CTIs. Differences in switch costs between mappings were small and nonsignificant in all experiments except for Experiment 4 (see Table 1). However, when all experiments were analyzed together, we did obtain a significant 24-ms difference in switch costs. There was neither a consistent pattern of mapping-order effects across experiments (see Table 3 ) nor significant differences in switch costs between mappings in analyses restricted to first-session data. In all experiments, both mappings yielded significant reductions in switch cost across cue-target interval (see Table 4), and there were significant cue-switching effects with the 2:1 mapping (see Table 5). Modeling analyses indicated that our model of cue encoding fit the data from both mappings about equally well (see Table 6) and with similar parameter values except for Experiments 4 and 5 (see Table 7). Overall, performance was more similar than it was different between mappings, a point most evident in Figure 7, which shows mean cued RTs for both mappings plotted against each other for each combination of transition and CTI in each experiment. The data are clustered closely around the unity line, consistent with a significant correlation of .98 between mappings $(p<.001)$.

Our results differ from Altmann's (2006) in three major respects. First, whereas Altmann found that switch cost was 166-ms larger with a 2:1 mapping compared with a 1:1 mapping, we obtained no differences approaching that magnitude,

Table 3

Differences in Switch Costs by Session and Mapping

\begin{tabular}{|c|c|c|c|c|c|c|c|}
\hline \multirow[b]{2}{*}{ Experiment } & \multirow[b]{2}{*}{$N$} & \multicolumn{3}{|c|}{ Switch cost in first session } & \multicolumn{3}{|c|}{ Switch cost in second session } \\
\hline & & 2:1 mapping & 1:1 mapping & Difference & 2:1 mapping & 1:1 mapping & Difference \\
\hline 1 & 16 & 110 & $81(85)$ & $29(25)$ & 60 & 62 & -2 \\
\hline 2 & 16 & 119 & $147(156)$ & $-28(-37)$ & 113 & 74 & 39 \\
\hline 3 & 16 & 203 & $204(240)$ & $-1(-37)$ & 174 & 139 & 35 \\
\hline 4 & 16 & 263 & $181(189)$ & $82(74)$ & 181 & 159 & 22 \\
\hline 5 & 16 & 313 & 227 (274) & $86(39)$ & 163 & 189 & -26 \\
\hline Altmann (2006) & 15 & 360 & 194 & $166^{*}$ & & & \\
\hline
\end{tabular}

Note. $\quad N=$ number of subjects with data for each mapping. Even though mapping was manipulated within subjects in our experiments, it was analyzed as a between-subjects factor in these analyses (as in Altmann, 2006), which is why $N=16$ instead of 32 . Numbers in parentheses reflect first-session analyses restricted to data from the first half of the 1:1 mapping session. Switch costs are in milliseconds.

${ }^{*} p<.05$ for the Mapping $\times$ Transition interaction from the relevant analysis of variance. (For Altmann's data, we indicate the results of his statistical analyses.) 
Table 4

Changes in Switch Costs Across CTI by Mapping

\begin{tabular}{|c|c|c|c|c|c|c|c|}
\hline \multirow[b]{2}{*}{ Experiment } & \multirow[b]{2}{*}{$N$} & \multicolumn{3}{|c|}{ Switch cost with $1: 1$ mapping } & \multicolumn{3}{|c|}{ Switch cost with $2: 1$ mapping } \\
\hline & & Shortest CTI & Longest CTI & Difference & Shortest CTI & Longest CTI & Difference \\
\hline 1 & 32 & 82 & 35 & $47^{*}$ & 88 & 38 & $50^{*}$ \\
\hline 2 & 32 & 150 & 61 & $89 *$ & 128 & 73 & $55^{*}$ \\
\hline 3 & 32 & 210 & 99 & $111^{*}$ & 216 & 106 & $110^{*}$ \\
\hline 4 & 32 & 254 & 77 & $177^{*}$ & 293 & 118 & $175^{*}$ \\
\hline 5 & 32 & 246 & 170 & $76^{*}$ & 303 & 173 & $130^{*}$ \\
\hline Altmann (2006) & 15 & 210 & 177 & 33 & 409 & 312 & 97 \\
\hline
\end{tabular}

Note. $\quad N=$ number of subjects with data for each mapping; CTI = cue-target interval. Switch costs are in milliseconds.

${ }^{*} p<.05$ for the Omnibus Transition $\times$ CTI interaction (involving all CTIs) from the relevant analysis of variance. (For Altmann's data, we indicate the results of his statistical analyses.)

even though we had adequate statistical power to detect large differences. Our results suggest that Altmann's effect size is not representative, although further investigation of task differences may qualify this conclusion. Second, whereas Altmann found nonsignificant reductions in switch cost across CTI, we obtained significant reductions with both mappings in all experiments. The consistency of our results across several methodological changes suggests that the interaction is not as unreliable as Altmann opined. Third, whereas Altmann found that our model fit 2:1 mapping data better than it fit 1:1 mapping data, we obtained no clear evidence of a bias (see also Logan \& Bundesen, 2003), suggesting that Altmann's finding lacks generality. Our results did partially replicate Altmann's in one important respect: We obtained significant differences in switch costs between mappings in Experiment 4 and in the analysis of all experiments together. Although these differences were much smaller than what Altmann found, they were nevertheless present and warrant discussion.

\section{Explaining Differences Between Mappings}

Altmann (2006) suggested two explanations for differences in switch costs between mappings. First, he suggested the "increase in task-switch cost might reflect effects of cue-task mapping on the probability of actually switching tasks" (Altmann, 2006, p. 1021). Although switch costs are modulated by the probability of task switching (Monsell \& Mizon, 2006; Schneider \& Logan, 2006), it is unclear how this explanation would apply in the present context. Cues are typically selected randomly across trials, making the probability of task switching equal to .50 regardless of the mapping. More specifically, with a 1:1 mapping, task switches and cue repetitions each occur with a probability of .50 , and with a $2: 1$ mapping, task switches, task repetitions, and cue repetitions occur with probabilities of $.50, .25$, and .25 , respectively. However, not all switch probabilities are equated between mappings. For example, the probability that a cue switch signals a task switch does differ between mappings (being 1.00 and .67 with $1: 1$ and 2:1 mappings, respectively), but it is still the case that the unconditional probability of a task switch does not. ${ }^{8}$ Thus, it is unclear how the probability of task switching would explain differences between mappings.

Altmann's (2006) second suggestion was that the "increase in task-switch cost . . . might also reflect the need for a new layer of processing or representation to cope with the more complex cuetask mapping" (Altmann, 2006, p. 1021). However, he did not discuss the nature of the new layer of processing or why it would specifically inflate switch cost as opposed to having a generic effect such as prolonging overall RT. Moreover, it is unclear whether adding more cue-task mappings increases the complexity of any particular cue-task mapping. Regardless of the mapping, a given cue is only ever associated with one task, so its complexity as a retrieval cue never changes. In the absence of some specification of the new layer of processing evoked by extra cue-task mappings, this explanation remains vague.

The idea that a new layer of processing is evoked by a $2: 1$ mapping is subject to a deeper criticism. The idea rests on the inference that a performance difference between mappings corresponds to a qualitative difference in processing. However, this need not be the case. A performance difference could reflect a quantitative difference in the same processing for both mappings. For example, the time it takes to process a cue could depend on the number of potentially relevant cues (consistent with our model's parameter values for Experiments 4 and 5; see Table 7) without cue processing being qualitatively different between mappings. We contend that performance differences between mappings are problematic only if they reflect qualitative differences in processing. If processing merely differs quantitatively, then the use of a 2:1 mapping would be appropriate for making inferences about performance with a $1: 1$ mapping. Effect sizes might differ between mappings, but the mechanisms underlying the effects would not, so a manipulation that affects switch cost with a 2:1 mapping should affect switch cost with a 1:1 mapping in a similar way. This was the case with our CTI manipulation: There were significant reductions in switch cost across CTI with both mappings in all our experiments (and these effects were not modulated by higher order interactions with mapping or mapping order; see Table 2). However, neither Altmann's (2006) data nor our data can resolve the quantitative versus qualitative processing issue. In all our experiments there was a numerical trend for slightly larger switch costs with a 2:1 mapping. These small effect sizes suggest a quantitative change in processing rather than the

\footnotetext{
${ }^{8}$ One way to control the probability that a cue switch signals a task switch is to prevent cue repetitions (Monsell \& Mizon, 2006).
} 
Table 5

Task-Switching and Cue-Switching Effects With the 2:1 Mapping

\begin{tabular}{lcccccc}
\hline Experiment & $N$ & Task switches & Task repetitions & Cue repetitions & Task-switching effect & Cue-switching effect \\
\hline 1 & 32 & 753 & 717 & 668 & $36^{*}(-6)$ & $49^{*}\left(56^{\dagger}\right)$ \\
2 & 32 & 884 & 836 & 768 & $48^{*}(22)$ & $(32)$ \\
3 & 32 & 1,049 & 1,009 & 860 & $40(-22)$ & $149^{*}(131)$ \\
4 & 32 & 974 & 925 & 752 & $9^{*}\left(32^{\dagger}\right)$ & $173^{*}\left(143^{\dagger}\right)$ \\
5 & 32 & 980 & 892 & 742 & $8^{*}(9)$ & 74 \\
Altmann (2006) & 15 & 1,364 & 1,290 & 1,003 & $280^{*}\left(121^{\dagger}\right)$ \\
\hline
\end{tabular}

Note. $\quad N=$ number of subjects with data for each mapping; Task-switching effect $=$ difference between task switches and task repetitions; Cue-switching effect $=$ difference between task repetitions and cue repetitions. Values in parentheses indicate the differences in each transition effect between the shortest and longest CTIs. Values are in milliseconds.

${ }^{\dagger} p<.05$ for the Omnibus Transition $\times$ CTI interaction (involving all CTIs) from the relevant analysis of variance (ANOVA) involving only the transitions of interest. $" p<.05$ for the contrast using the error term for the main effect of transition from the relevant ANOVA. (For Altmann's data, we indicate the results of his statistical analyses.)

addition of a new layer of processing, for it seems implausible that a new process would take a mere $24 \mathrm{~ms}$ (on average) to finish. Nevertheless, the source(s) of performance differences between mappings remains an issue that should be addressed in future research.

\section{Benefits of Using a 2:1 Mapping}

Even if further research were to establish larger performance differences between mappings, we think there are benefits to using a 2:1 mapping. First, it is still the case that a 2:1 mapping allows estimation of task-switching effects with a constant cue transition. Whether these effects correspond directly to the latent taskswitching effects present with a 1:1 mapping is unclear due to uncertainty about processing differences between mappings (as discussed earlier), but it could be interesting to study these effects in their own right.

A second benefit is that the use of a 2:1 mapping has revealed reliable cue-switching effects with a constant task transition, as demonstrated in the present study (see Table 5) and in previous studies (e.g., Logan \& Bundesen, 2003; Mayr \& Kliegl, 2003; Monsell \& Mizon, 2006). Cue-switching effects not only imply that the confound between task switching and cue switching with a 1:1 mapping is a real problem, but they can be used to study how cues are processed. Cue-switching effects have been used to identify when cue encoding has finished (Arrington et al., 2007), to distinguish between perceptual priming and control processing of cue representations (Grange \& Houghton, 2010), to determine the nature of the representation guiding the performance of memorized task sequences (Mayr, in press), and to constrain models of cue encoding (Logan \& Bundesen, 2003; Schneider \& Logan, 2005, 2006).

A third benefit of using a 2:1 mapping is that it can provide insight into cognitive processes by ruling out cue-related effects. One example is the case of task-set inhibition (Mayr \& Keele, 2000). When switching among three tasks, RT is longer for Lag 2 task repetitions than for Lag 2 task switches. This Lag 2 repetition cost is thought to reflect inhibition when switching tasks, but it is unclear what is inhibited-the locus of inhibition could be abstract task representations, cues, targets, responses, or some combination thereof. Use of a 2:1 mapping indicated that the inhibition is not uniquely linked to cues because Lag 2 repetition cost was observed with Lag 2 cue repetitions and Lag 2 cue switches (Altmann, 2007; Gade \& Koch, 2008). There are likely other contexts in which a 2:1 mapping may prove useful

Table 6

Model Fit Indices

\begin{tabular}{|c|c|c|c|c|c|}
\hline \multirow[b]{2}{*}{ Experiment } & \multirow[b]{2}{*}{ Fit } & \multicolumn{2}{|c|}{ 1:1 mapping } & \multicolumn{2}{|c|}{ 2:1 mapping } \\
\hline & & RMSD & $r$ & RMSD & $r$ \\
\hline \multirow[t]{2}{*}{1} & Individual & 27 & .941 & 34 (37) & $.926(.924)$ \\
\hline & Group & 9 & .995 & $14(13)$ & $.988(.990)$ \\
\hline \multirow[t]{2}{*}{2} & Individual & 51 & .913 & $56(68)$ & $.901(.862)$ \\
\hline & Group & 10 & .996 & $13(15)$ & $.993(.991)$ \\
\hline \multirow[t]{2}{*}{3} & Individual & 68 & .898 & 87 (105) & $.879(.842)$ \\
\hline & Group & 12 & .996 & $28(23)$ & $.982(.987)$ \\
\hline \multirow[t]{2}{*}{4} & Individual & 50 & .923 & $74(84)$ & $.890(.873)$ \\
\hline & Group & 10 & .997 & 20 (19) & $.993(.993)$ \\
\hline \multirow[t]{2}{*}{5} & Individual & 38 & .943 & $29(38)$ & $.958(.945)$ \\
\hline & Group & 42 & .953 & $22(18)$ & $.993(.995)$ \\
\hline
\end{tabular}

Note. $\quad$ RMSD $=$ root-mean-square deviation between data and model predictions (in milliseconds); $r=$ correlation between data and model predictions. Values in parentheses are from fits to the 2:1 mapping data, including task repetitions. 
Table 7

Best-Fitting Model Parameters

\begin{tabular}{|c|c|c|c|c|c|c|c|c|}
\hline \multirow[b]{2}{*}{ Experiment } & \multirow[b]{2}{*}{ Fit } & \multicolumn{3}{|c|}{ 1:1 mapping } & \multicolumn{4}{|c|}{ 2:1 mapping } \\
\hline & & $R T_{\text {Base }}$ & $\mu_{\mathrm{TS}}$ & $\mu_{\mathrm{CR}}$ & $R T_{\text {Base }}$ & $\mu_{\mathrm{TS}}$ & $\mu_{\mathrm{TR}}$ & $\mu_{\mathrm{CR}}$ \\
\hline \multirow[t]{2}{*}{1} & Individual & $603 \pm 41$ & $275 \pm 32$ & $177 \pm 26$ & $\begin{array}{c}595 \pm 56 \\
(588 \pm 50)\end{array}$ & $\begin{array}{c}294 \pm 43 \\
(303 \pm 44)\end{array}$ & $(259 \pm 44)$ & $\begin{array}{c}177 \pm 29 \\
(185 \pm 35)\end{array}$ \\
\hline & Group & 610 & 270 & 171 & $\begin{array}{c}604 \\
(601)\end{array}$ & $\begin{array}{c}288 \\
(292)\end{array}$ & (249) & $\begin{array}{c}170 \\
(174)\end{array}$ \\
\hline \multirow[t]{2}{*}{2} & Individual & $668 \pm 44$ & $414 \pm 90$ & $276 \pm 48$ & $\begin{array}{c}652 \pm 39 \\
(648 \pm 42)\end{array}$ & $\begin{array}{c}390 \pm 54 \\
(394 \pm 57)\end{array}$ & $(335 \pm 52)$ & $\begin{array}{c}240 \pm 42 \\
(244 \pm 46)\end{array}$ \\
\hline & Group & 698 & 388 & 245 & $\begin{array}{c}667 \\
(668)\end{array}$ & $\begin{array}{c}378 \\
(377)\end{array}$ & (318) & $\begin{array}{c}227 \\
(226)\end{array}$ \\
\hline \multirow[t]{2}{*}{3} & Individual & $735 \pm 82$ & $447 \pm 75$ & $225 \pm 48$ & $\begin{array}{c}730 \pm 74 \\
(718 \pm 78)\end{array}$ & $\begin{array}{c}497 \pm 67 \\
(510 \pm 77)\end{array}$ & $(460 \pm 76)$ & $\begin{array}{c}252 \pm 55 \\
(263 \pm 71)\end{array}$ \\
\hline & Group & 761 & 424 & 200 & $\begin{array}{c}759 \\
(762)\end{array}$ & $\begin{array}{c}468 \\
(464)\end{array}$ & (418) & $\begin{array}{c}230 \\
(226)\end{array}$ \\
\hline \multirow[t]{2}{*}{4} & Individual & $698 \pm 80$ & $421 \pm 60$ & $183 \pm 48$ & $\begin{array}{c}610 \pm 65 \\
(593 \pm 65)\end{array}$ & $\begin{array}{c}544 \pm 87 \\
(561 \pm 107)\end{array}$ & $(504 \pm 91)$ & $\begin{array}{c}266 \pm 65 \\
(286 \pm 82)\end{array}$ \\
\hline & Group & 721 & 400 & 166 & $\begin{array}{c}643 \\
(650)\end{array}$ & $\begin{array}{c}519 \\
(511)\end{array}$ & (452) & $\begin{array}{c}237 \\
(229)\end{array}$ \\
\hline \multirow[t]{2}{*}{5} & Individual & $706 \pm 68$ & $464 \pm 91$ & $177 \pm 37$ & $\begin{array}{c}613 \pm 69 \\
(580 \pm 86)\end{array}$ & $\begin{array}{c}617 \pm 153 \\
(653 \pm 179)\end{array}$ & $(540 \pm 158)$ & $\begin{array}{l}296 \pm 89 \\
(336 \pm 120)\end{array}$ \\
\hline & Group & 725 & 452 & 161 & $\begin{array}{c}660 \\
(663)\end{array}$ & $\begin{array}{c}590 \\
(586)\end{array}$ & $(475)$ & $\begin{array}{c}256 \\
(252)\end{array}$ \\
\hline Altmann (2006) & Individual & 833 & 494 & 242 & 684 & 1,005 & & 546 \\
\hline
\end{tabular}

Note. $R T_{\text {Base }}=$ residual processing time; $\mu=$ mean cue encoding time for task switches (TS), task repetitions (TR), and cue repetitions (CR). Means with $95 \%$ confidence intervals are reported for the individual fits. Values in parentheses are from fits to the 2:1 mapping data, including task repetitions. Altmann's parameter values are from his reported model fits. All parameter values are in milliseconds.

for isolating cue-based contributions to task-switching phenomena.

\section{Concluding Remarks}

We invite readers to draw their own conclusions, but our results suggest to us that the use of a 2:1 mapping is a viable solution to the problematic confound that is inherent with a $1: 1$ mapping. However, the solution is not without limitations, the main one being that we did obtain small but reliable differences in switch costs between mappings in two of our analyses. Given that these effects may reflect only quantitative differences in processing, we think it would be premature to reject the 2:1 mapping without further research. Moreover, regardless of the final verdict on the 2:1 mapping, we think it is important not to lose sight of the overarching issue, which is the confound that exists with a 1:1 mapping. Being cognizant of the problem and
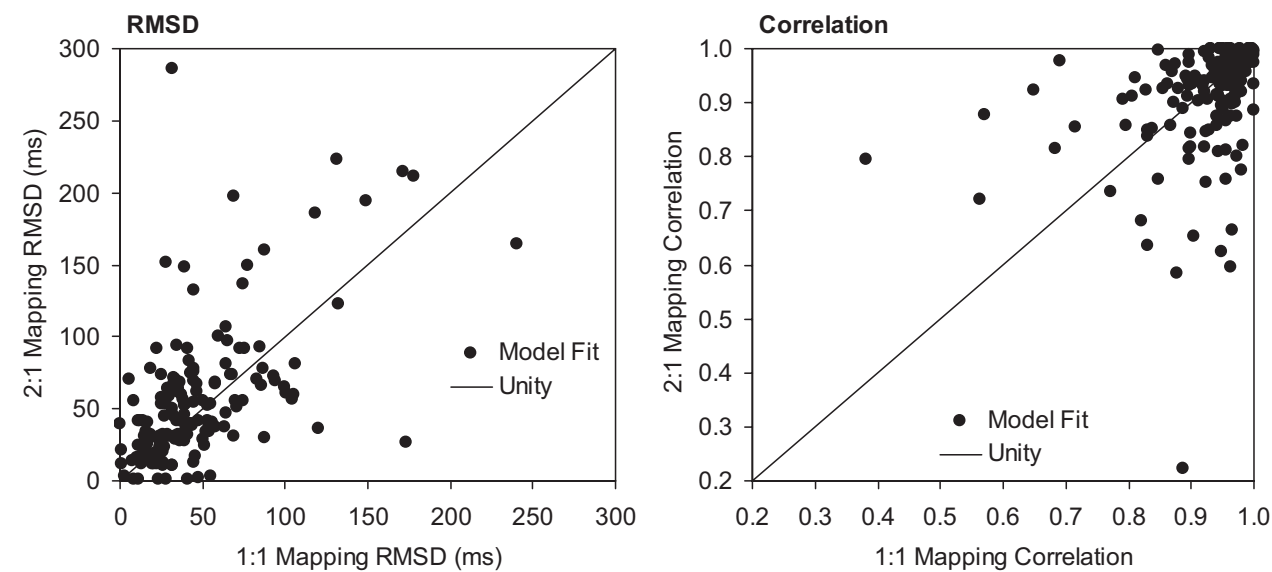

Figure 6. Corresponding root-mean-square deviation (RMSD) and correlation values for individual-subject model fits. Each RMSD and correlation is between an individual subject's data and the model fit to that data. Each point in the figure is the relevant fit index (RMSD or correlation) for a given subject in the 1:1 mapping condition plotted against the corresponding fit index for that subject in the 2:1 mapping condition. 


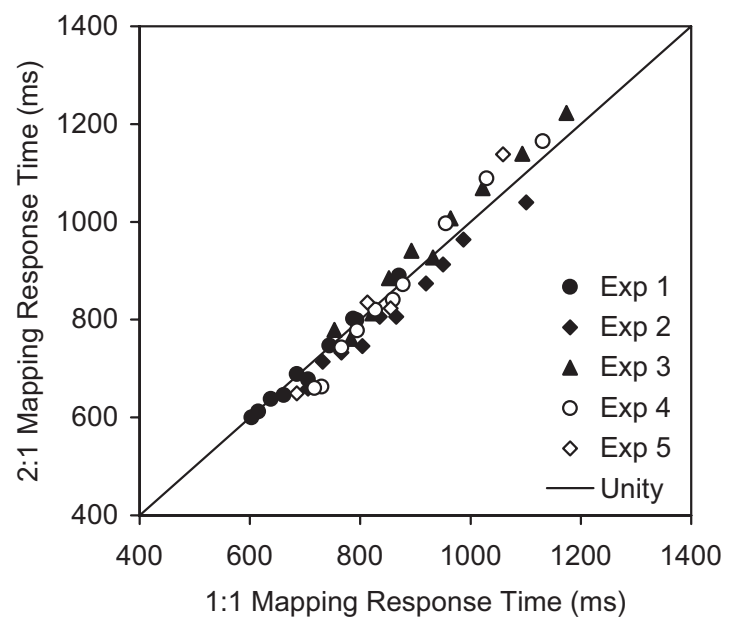

Figure 7. Corresponding mean response times with the 1:1 and 2:1 mappings for each combination of transition (task switch or cue repetition) and cue-target interval in each experiment (Exp), ignoring task repetitions.

attempting to address it—whether it be with a 2:1 mapping or by some other means-will lead to better inferences about the cognitive mechanisms involved in task switching.

\section{References}

Altmann, E. M. (2004). The preparation effect in task switching: Carryover of SOA. Memory \& Cognition, 32, 153-163.

Altmann, E. M. (2006). Task switching is not cue switching. Psychonomic Bulletin \& Review, 13, 1016-1022.

Altmann, E. M. (2007). Cue-independent task-specific representations in task switching: Evidence from backward inhibition. Journal of Experimental Psychology: Learning, Memory, and Cognition, 33, 892-899. doi:10.1037/0278-7393.33.5.892

Arrington, C. M., Altmann, E. M., \& Carr, T. H. (2003). Tasks of a feather flock together: Similarity effects in task switching. Memory \& Cognition, 31, 781-789.

Arrington, C. M., Logan, G. D., \& Schneider, D. W. (2007). Separating cue encoding from target processing in the explicit task-cuing procedure: Are there "true" task switch effects? Journal of Experimental Psychology: Learning, Memory, and Cognition, 33, 484-502. doi:10.1037/ 0278-7393.33.3.484

Faul, F., Erdfelder, E., Lang, A.-G., \& Buchner, A. (2007). G*Power 3: A flexible statistical power analysis program for the social, behavioral, and biomedical sciences. Behavior Research Methods, 39, 175-191.

Gade, M., \& Koch, I. (2008). Dissociating cue-related and task-related processes in task inhibition: Evidence from using a 2:1 cue-to-task mapping. Canadian Journal of Experimental Psychology, 62, 51-55. doi:10.1037/1196-1961.62.1.51
Grange, J. A., \& Houghton, G. (2010). Cue-switch costs in task-switching: Cue priming or control processes? Psychological Research, 74, 481490. doi:10.1007/s00426-009-0270-y

Koch, I. (2008). Instruction effects in task switching. Psychonomic Bulletin \& Review, 15, 448-452. doi:10.3758/PBR.15.2.448

Logan, G. D., \& Bundesen, C. (2003). Clever homunculus: Is there an endogenous act of control in the explicit task-cuing procedure? Journal of Experimental Psychology: Human Perception and Performance, 29, 575-599. doi:10.1037/0096-1523.29.3.575

Logan, G. D., Schneider, D. W., \& Bundesen, C. (2007). Still clever after all these years: Searching for the homunculus in explicitly cued task switching. Journal of Experimental Psychology: Human Perception and Performance, 33, 978-994. doi:10.1037/0096-1523.33.4.978

Mayr, U. (2010). The surface structure and the deep structure of sequential control: What can we learn from task-span switch costs? Psychonomic Bulletin \& Review, 17, 693-698. doi:10.3758/PBR.17.5.693

Mayr, U., \& Keele, S. W. (2000). Changing internal constraints on action: The role of backward inhibition. Journal of Experimental Psychology: General, 129, 4-26. doi:10.1037/0096-3445.129.1.4

Mayr, U., \& Kliegl, R. (2003). Differential effects of cue changes and task changes on task-set selection costs. Journal of Experimental Psychology: Learning, Memory, and Cognition, 29, 362-372. doi:10.1037/02787393.29.3.362

Meiran, N. (1996). Reconfiguration of processing mode prior to task performance. Journal of Experimental Psychology: Learning, Memory, and Cognition, 22, 1423-1442. doi:10.1037/0278-7393.22.6.1423

Meiran, N., Chorev, Z., \& Sapir, A. (2000). Component processes in task switching. Cognitive Psychology, 41, 211-253. doi:10.1006/ cogp. 2000.0736

Monsell, S. (2003). Task switching. Trends in Cognitive Sciences, 7, 134-140. doi:10.1016/S1364-6613(03)00028-7

Monsell, S., \& Mizon, G. A. (2006). Can the task-cuing paradigm measure an endogenous task-set reconfiguration process? Journal of Experimental Psychology: Human Perception and Performance, 32, 493-516. doi:10.1037/0096-1523.32.3.493

Schneider, D. W., \& Logan, G. D. (2005). Modeling task switching without switching tasks: A short-term priming account of explicitly cued performance. Journal of Experimental Psychology: General, 134, 343-367. doi:10.1037/0096-3445.134.3.343

Schneider, D. W., \& Logan, G. D. (2006). Priming cue encoding by manipulating transition frequency in explicitly cued task switching. Psychonomic Bulletin \& Review, 13, 145-151.

Schneider, D. W., \& Logan, G. D. (2009). Selecting a response in task switching: Testing a model of compound cue retrieval. Journal of Experimental Psychology: Learning, Memory, and Cognition, 35, 122136. doi:10.1037/a0013744

Sudevan, P., \& Taylor, D. A. (1987). The cuing and priming of cognitive operations. Journal of Experimental Psychology: Human Perception and Performance, 13, 89-103. doi:10.1037/0096-1523.13.1.89

Received March 22, 2010

Revision received October 4, 2010

Accepted October 7, 2010 